\title{
Martin Hess
}

Die Anwendbarkeit des humanitären Völkerrechts, insbesondere in gemischten Konflikten Schulthess, Zürich, 1985, Schweizer Studien zum internationalen Recht, Band 39, 286 S., sfr 45 ,-

In einer unfriedlichen Welt, deren bewaffnete Konflikte sich großenteils nicht mehr säuberlich als "international" oder "nicht-international" kategorisieren lassen, in der aber auch erstmals der Versuch unternommen wurde, ein Mindestmaß humanitären Verhaltens auch für nicht-internationale Konflikte völkerrechtlich zu normieren, liegt die Notwendigkeit einer Untersuchung darüber auf der Hand, welche Normen in welchen Konflikten anwendbar sind und angewendet werden. Dieser Aufgabe unterzieht sich mit großer Akribie (allein das Inhaltsverzeichnis umfaßt mehr als sieben Druckseiten!) Martin Hess in seiner hier zu besprechenden von Dietrich Schindler betreuten Zürcher Dissertation.

Der Autor beginnt mit einer Darstellung des geltenden "Haager" und "Genfer" Rechts und seiner Fortentwicklung nach dem Zweiten Weltkrieg und kommt zu der zunächst erschreckenden Feststellung, daß auf Grund des in der Wiener Vertragsrechtskonvention von 1978 anerkannten "clean slate"-Prinzips für die (Nicht-)Ubernahme vertragsrechtlicher Verpflichtungen durch im Wege der Entkolonialisierung unabhängig gewordene Staaten das "klassische" Kriegsrecht nur noch für eine Minderheit der Staaten gilt, so die Haager LKO von 1907 für 37. Eine Ausnahme bildet nur das Giftgasprotokoll von 1925, das immerhin für 105 Staaten verbindlich ist, wobei die späte Ratifizierung durch die USA (1975) auffällt. Inwieweit die Normen dieser Verträge auch für Nichtvertragsparteien als Gewohnheitsrecht verbindlich sind, ist strittig und für die einzelnen Geund Verbote unterschiedlich zu beurteilen. Demgegenüber würdigt er das Bestreben der Völkerrechtsgemeinschaft, humanitäre Regeln für alle bewaffneten Konflikte verbindlich zu machen, ob es sich um erklärte Kriege handelt oder nicht. Hier sind in erster Linie die vier Genfer Konventionen von 1949 über

- Kranke und Verwundete im Felde (VKA),

- Kranke, Verwundete und Schiffbrüchige zur See (VKS),

- Kriegsgefangene (KGA) und

- den Schutz von Zivilpersonen in Kriegszeiten (ZPA)

mit den beiden Zusatzprotokollen von 1977 über den Schutz der Opfer internationaler (ZP I) und nichtinternationaler (ZP II) bewaffneter Konflikte zu nennen, aber auch das Haager Abkommen von 1954 über den Schutz von Kulturgut (HAK) und das Ubereinkommen von 1980 über das Verbot oder die Beschränkung des Einsatzes bestimmter konventioneller Waffen (CAC). Um nämlich eine universelle Anwendbarkeit zu ermöglichen, sehen die Genfer Konventionen auch den ad-hoc-Beitritt von nichtstaatlichen "Konfliktsparteien" vor (gemeinsamer Art. 2 Abs. 3), worunter nach Hess nicht nur Befreiungsbewegungen, sondern auch sonstige nicht anerkannte Regierungen und Widerstandsbewegungen verstanden werden müssen. Uber diese Ausweitung des Begriffs des internationalen Konflikts hinaus aber normiert der gemeinsame Art. 3 der Konven- 
tionen einen humanitären Mindeststandard auch für nichtinternationale Konflikte, der im ZP II konkretisiert wird. Eine vergleichbare Norm findet sich in Art. 19 HAK, während das CAC ausdrücklich nur für internationale Konflikte gilt.

Hier kommt der Verfasser auf sein Hauptproblem: Die Abgrenzungsschwierigkeiten zwischen internationalen und nichtinternationalen bewaffneten Konflikten und die rechtliche Einordnung der immer häufiger werdenden gemischten Konflikte, die durch Intervention dritter Staaten in einen nichtinternationalen Konflikt entstehen können, aber auch dadurch, daß ein Angriff von außen einen internen Konflikt erst auslöst. Für die Zeit von 1950 bis 1983 zählt er allein 14 solcher gemischter Konflikte auf! Da alle Versuche einer vertraglichen Regelung dieses Konfliktstyps scheiterten - der Vorschlag des IKRK, die für internationale Konflikte geltenden Regeln für anwendbar zu erklären, fand keine Mehrheit; der indonesische Vorschlag, trotz ausländischer Intervention einen nichtinternationalen Charakter anzunehmen, blieb isoliert - bringt Hess die von seinem Lehrer Schindler vertretene Komponententheorie ins Spiel. Hiernach ist ein gemischter Konflikt in seine rechtlich gesondert zu beurteilenden Komponenten zu zerlegen: Der Konflikt zwischen etablierter Regierung und Aufständischen bleibt demnach stets nichtinternational. Eine Intervention auf Seiten der Regierung ändert an diesem Charakter nichts, da dem Interventen kein Völkerrechtssubjekt gegenübersteht, mit dem er einen internationalen Konflikt führen könnte. Eine Intervention auf Seiten der Aufständischen aber internationalisiert den Konflikt insofern, als der Intervent mit der (noch) etablierten Regierung in einen internationalen Konflikt gerät. Ein Konflikt zwischen zwei Interventen auf verschiedenen Seiten hat auf jeden Fall internationalen Charakter. Als Maßstab für die Beurteilung, wer etabliert ist und wer nicht, läßt der Verfasser wohl mit Recht nur die Effektivität der Kontrolle über Bevölkerung und Staatsgebiet gelten, nicht aber, wie von einem Teil der Lehre gefordert, die Intensität der Kämpfe. Konsequenterweise ändert sich die rechtliche Qualifikation einer Intervention dann, wenn Aufständische die effektive Kontrolle gewinnen und ihrerseits etablierte Regierung werden. Gleiches gilt dann auch für die Rechtsstellung des - erfolgreichen oder erfolglosen Widerstands gegen eine von einem Interventen eingesetzte Marionettenregierung. Auf die völkerrechtliche Anerkennung kann es dann nicht mehr ankommen, da diese oft von politischem Zweckmäßigkeitsdenken bestimmt wird (vgl. Afghanistan auf der einen, Kampuchea auf der anderen Seite).

Der Verfasser räumt selbst ein, zu welch unbefriedigenden Ergebnissen eine solche Betrachtungsweise of t (meist?) führen kann. Er hält sie aber wohl zu Recht für das einzige Mittel, die chaotischen Fakten ordnend überblicken zu können. Sei der Überblick aber gewonnen, müsse man auch die Komponententheorie wieder überwinden, da sie sich im Sinne von Umberto Eco (Der Name der Rose) als "zwar nützlich aber unsinnig" erwiesen habe. Er zitiert hier den auch im italienischen Original mittelhochdeutsch stehenden Satz: "Er muoz gelîchesame die leiter abewerfen, sô er an ir ufgestigen ist."

Zuvor aber analysiert der Verfasser an Hand der aufgezeigten Kriterien fünf aktuelle gemischte Konflikte, wobei er in drei Fällen zu vorhersehbaren Ergebnissen kommt: 
- Afghanistan: Sowjetische Intervention zugunsten der - wenn auch erst von ihr selbst eingesetzten - etablierten Regierung, mithin kein internationaler Konflikt, zumal der Widerstand trotz weitgehend effektiver Kontrolle wegen seiner Zerstrittenheit keine international handlungsfähigen Organe bilden konnte. Anwendbar ist der gemeinsame Art. 3 der Genfer Konventionen, nicht dagegen das ZP II, da weder die Sowjetunion noch Afghanistan es ratifiziert haben. Art. 19 HAK muß nur von der Sowjetunion beachtet werden, da Afghanistan nicht Vertragspartei ist. In der Praxis wird allerdings auch Art. 3 a.a.O. kaum beachtet.

- Angola: Intervention Cubas auf Seiten der "etablierten" MPLA-Regierung, Südafrikas auf Seiten der "aufständischen" UNITA (der nebenherlaufende Namibia-Konflikt zwischen Südafrika und der von Angola aus operierenden SWAPO bleibt hier außer Betracht). Angola ist mangels Nachfolgeerklärung an keinen Vertrag des humanitären Völkerrechts gebunden. Nur für Kampfhandlungen zwischen Cubanern und Südafrikanern gelten die Genfer und Haager Konventionen, für alle anderen Auseinandersetzungen (Regierung ./. UNITA, Regierung ./. Südafrika und Cuba ./. UNITA) dagegen nur Art. 3 a.a.O.

- Kampuchea: Intervention Vietnams gegen die damals etablierte Regierung Pol Pot, Einsetzung einer Marionettenregierung, die aber mangels eigener Machtmittel nicht als etabliert angesehen werden kann. Ebensowenig kann trotz Anerkennung durch die VN-Mehrheit die antivietnamesische Koalitionsregierung unter Prinz Sihanouk als etabliert angesehen werden, da sie nicht über nennenswertes Territorium verfügt. Somit ist die Besetzung durch Vietnam nach wie vor eine kriegeris̀che und dieses sowohl in seinem Verhältnis zu den Streitkräften der Koalitionsregierung wie zur Zivilbevölkerung an die vier Genfer Konventionen gebunden. Das ZP I gilt dagegen nicht, da Kampuchea nicht Vertragspartei ist. Zwischen beiden Regierungen Kampucheas gelten Art. 3 a.a.O. und Art. 19 HAK, nicht dagegen ZP II, dem Kampuchea nicht beigetreten ist.

Zu einer "Null-Lösung" kommt der Verfasser auch für die Konflikte im Libanon und im Tschad, da in beiden Ländern die von der überwiegenden Mehrheit der Völkerrechtsgemeinschaft anerkannte Regierung nicht mehr als effektiv angesehen werden könne, so daß nur noch von jeweils anderen Interventen unterstützte Bürgerkriegsparteien vorhanden seien. Dies führt zu einer völlig verworrenen und verwirrenden Rechtslage:

- Libanon: Sowohl Syrien als auch Israel sind kriegerische Besatzer, die im Konflikt miteinander an das gesamte humanitäre Völkerrecht gebunden sind im Verhältnis zur libanesischen (und palästinensischen) Zivilbevölkerung an das ZPA. Im Verhältnis zu den Kombattanten der libanesischen Konfliktsparteien gelten nur Art. 3 a.a.O. und Art. 19 HAK. Gleiches gilt nach Ansicht des Verfassers auch für das Verhältnis Israel ./. PLO, obwohl diese eine weitgehend anerkannte Befreiungsorganisation ist. Die Rolle der amerikanischen und französischen Kontingente der multinationalen Friedentruppe ist während ihres Eingreifens in die Kämpfe ebenso zu beurteilen wie die der Syrer und Israelis. Die britischen und italienischen Kontingente wurden dagegen nicht zur Bürgerkriegspartei. 
- Tschad: Trotz gegenteiliger Stellungnahmen der Interventen kann weder das Eingreifen Frankreichs und Zaires zugunsten Oueddeis noch das Libyens zugunsten Habrés als Unterstützung einer etablierten Regierung angesehen werden. Alle drei sind in einen internationalen Konflikt mit dem Staat Tschad verwickelt, der sie zur Beachtung des ZPA verpflichtet. Die übrigen Genfer Abkommen gelten mangels handlungsfähiger tschadischer Staatsorgane nicht. Der tschadische Bürgerkrieg ist nicht internationalen Charakters, zu Auseinandersetzungen zwischen den Interventen ist es nicht gekommen.

Es ist nur zu verständlich, daß der Verfasser angesichts der Unübersichtlichkeit der Ergebnisse die fehlende Gemeinverständlichkeit des geltenden humanitären Völkerrechts beklagt. So fordert er die Rückkehr zu den humanitären Grundwerten, wie sie das IKRK in seinen "Règles fondamentales du droit international humanitaire applicables dans les conflits armés" formuliert hat.

Diesem Appell muß man voll zustimmen. Allerdings bleibt auch die Skepsis angebracht, die in dem vom Verfasser seiner Arbeit vorangestellten Gottfried Keller Zitat zum Ausdruck kommt: Denn wo die Zeiten ineinanderströmen und die Leidenschaften, die reinen wie die unreinen, darauf einherfahren, sind die Rechtsleute schwache Dammwächter. - Leider.

Karl Leuteritz

\section{Mohan Krischke Ramaswamy}

Ethnologie für Anfänger - eine Einführung aus entwicklungspolitischer Sicht

Westdeutscher Verlag Opladen, 1985, DM 25,80

Untertitel und Inhaltsabriß auf der letzten Einbandseite dieses neuen Taschenbuches machen neugierig: "Ethnologie für Anfänger" sei eine "andere" Einführung in das Fach, eine Präsentation aus entwicklungspolitischer Sicht. Der Uberblick wende sich nicht nur an Studienanfänger, sondern vor allem an Personen, die sich waus den verschiedensten beruflichen Gründen - zum Beispiel als Entwicklungshelfer - mit fremder Kultur beschäftigen". Diese Sätze scheinen eher vom Verlag als vom Autor selbst formuliert worden zu sein. Die Lektüre hält nämlich nicht, was der Untertitel verspricht.

Als Einstieg in eine Wissenschaft von Menschen und ihren Daseinsformen wählt Ramaswamy den gefährlichen Weg über das Fachvokabular. Seine Ausführungen über Arbeitsgebiete, Geschichte, Forscher, Schulen, theoretische Ansätze, Methoden und Probleme der Ethnologie ranken sich zu eng an Definitionen und Interpretationen von Fachausdrücken empor. Hinter dieser Vorgehensweise mag das Bemühen um sprachliche Klarheit und/oder die Absicht stecken, den Studienanfänger mit der Nomenklatur seines Faches vertraut zu machen. Doch durch den Verzicht auf Fallbeispiele und Fotos gerät dieser Ansatz zu einem luftabschnürenden Begriffskorsett. 